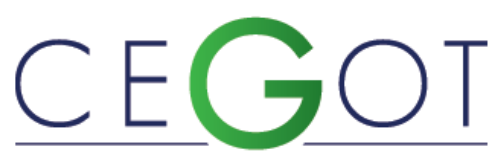

Centro de Estudos de Geografia e Ordenamento do Território
PARFITT, CLAURE MORRONE

Universidade Federal de Pelotas (UFPEL)

Centro de Integração do MERCOSUL

9602-080 Pelotas RS, Brasil

clauremparfitt@gmail.com

DE MATTOS, VIVIANE LEITE DIAS

Universidade Federal do Rio Grande (FURG)

Instututo de Matemática Estatística e Física

96203-900 Rio Grande, RS, Brasil

viviane.leite.mattos@gmail.com

\title{
AVALIANDO O DESEMPENHO DE PLANO DIRETOR DE DESENVOLVIMENTO URBANO NA PRODUÇÃO DO ESPAÇO EM ÁREAS DE PRESERVAÇÃO AMBIENTAL
}

Referência: Parfitt, Claure Morrone; De Mattos, Viviane Leite Dias (2021). AVALIANDO O DESEMPENHO DE PLANO DIRETOR DE DESENVOLVIMENTO URBANO NA PRODUÇÃO DO ESPAÇO EM ÁREAS DE PRESERVAÇÃO AMBIENTAL. Revista de Geografia e Ordenamento do Território (GOT), no 22 (Dezembro). Centro de Estudos de Geografia e Ordenamento do Território, p. 230 - 253, dx.doi.org/10.17127/got/2021.22.010

\section{RESUMO}

A tomada de decisão em planejamento urbano deve estar assentada em informações precisas sobre o desempenho dos espaços produzidos relacionados a aspectos socioeconômicos e ambientais. No entanto, apesar do corrente padrão observado nas áreas periurbanas da maioria das cidades brasileiras, o sistema de planejamento implementado não estabelece modelos de investigação dos conflitos decorrentes da produção do espaço urbano. Com o intuito de ampliar as formas de avaliação e controle de impactos ambientais, apresenta-se uma estrutura teórico-metodológica de avaliação de desempenho do espaço produzido em Áreas Urbanas de Preservação Ambiental, em que indicadores consistem em importantes medidas para identificar as discrepâncias internas ao sistema evidenciando a sua conformação. Este trabalho tem como objetivo avaliar o desempenho do Plano Diretor de Desenvolvimento Urbano de Pelotas (RS, Brasil) na produção do espaço em áreas de Preservação Ambiental. Na investigação foram considerados aspectos socioespaciais, socioambientais e socioeconômicos. Utilizam-se procedimentos estatísticos multivariados, que podem constituir a base de uma estrutura para o monitoramento e gestão do desenvolvimento de áreas de preservação em contexto urbano.

Palavras-chave: Planejamento urbano; Plano Diretor; Avaliação de desempenho; Estatística Multivariada. 


\section{ABSTRACT}

Decision-making in urban planning should be based on accurate information on the performance of the spaces produced related to socioeconomic and environmental aspects. However, despite the current pattern observed in the peri-urban areas of most Brazilian cities, the planning system implemented does not establish models for investigating conflicts arising from the production of urban space. To expand the forms of assessment and control of environmental impacts, a theoretical-methodological framework is presented for assessing the performance of the space produced in urban areas of environmental preservation. The indicators of that framework are important measures to identify internal discrepancies to the system, showing its heterogeneity. The present study aimed to assess the performance of the Master Plan for Urban Development of the city of Pelotas, RS, Brazil in the production of space in areas of Environmental Preservation. The investigation considered socio-spatial, socioenvironmental and socio-economic aspects. The proposed analysis uses multivariate statistical analysis to classify the space produced. The study is useful in the production of a framework for monitoring and managing the current and future panorama of development in urban environmental preservation areas.

Keywords: Urban planning, Master Plan Performance assessment, Multivariate Statistics, Master Plan.

\section{Introdução}

O processo de urbanização tem gerado problemas de considerável gravidade, concernentes ao uso e à ocupação do espaço. A convergência da população e inadequados padrões tecnológicos têm reforçado um ambiente urbano altamente degradado. A produção do espaço é estudada por autores como Harvey ( 1980 ) e Gottdiener(1997) porém recursos naturais e sistemas ecológicos são frequentemente desconsiderados.

Nesse sentido, de acordo com Shen et al., (2017) a degradação da qualidade ambiental é uma das maiores consequências da urbanização, bem como a expansão sobre florestas, banhados, ecossistemas e habitats ${ }^{1}$ (Seto et al., 2010). Outro problema é o crescimento periurbano e urban sprawl, ${ }^{2}$ esse último observado principalmente nas cidades norte-americanas (Martine, 2011).

\footnotetext{
${ }^{1}$ Habitat é o ambiente natural em que vive um organismo, ou o ambiente físico que envolve uma espécie.

2 Urban sprawl consiste em um padrão de expansão urbana de baixa densidade, espacialmente disperso, caracterizado por uso dominantemente habitacional e com desenvolvimento de faixas comerciais.
} 
Embora autores Calthorpe (1996) Duany et al (2010) considerem o sprawl como parcialmente sustentável, a medida que as cidades crescem há maior utilização de recursos naturais e emissão de gases de efeito estufa Sarkodie et al (2020), Ali et al (2021).

Sabe-se que a implantação de atividades em determinada localização pode exercer impactos ambientais, econômicos e sociais sobre o espaço, da mesma forma que Planos Diretores raramente têm êxito para orientar o desenvolvimento urbano (Sharifi et al., 2014). Nessa ótica, a implementação de sistemas de controle (monitoramento), se faz necessária, para que se obtenham séries de dados que permitam a identificação das alterações, assim como a avaliação e o auxílio à gestão urbana.

Nesse contexto, Yan et al. (2018) consideram que uma importante contribuição para o surgimento da forma urbana no debate do planejamento foi dada em 1990, com a introdução do conceito de Desenvolvimento Sustentável. Isto talvez tenha ocorrido por sua forte ligação com a forma da cidade, a dimensão física da sustentabilidade, sendo a forma urbana um dos elementos chave no impacto da urbanização sobre o meio ambiente (Seto et al., 2010).

Nessa seara, a prática de planejamento também é importante e complexa e sua avaliação um exercício necessário (Baer, 1997; Oliveira, 2010; Guyadeen \& Seasons, 2018; Hersperger et al. 2018).

Assim, o objetivo da atual política de desenvolvimento urbano criou a necessidade de utilização de procedimentos que avaliem a contribuição de soluções alternativas para os objetivos gerais de sustentabilidade Duany et al. (2010), Pupphachai (2017). Vários autores como Ye et al., (2011); Ali, (2015); Montgomery, B.; Dragićević, S. (2016); Raddad, (2016); Akbulut et al. (2018); Zambon et al. (2018) pesquisaram nesse sentido.

No entanto, apesar dos apelos orientados para o desempenho e o planejamento baseado em evidências, os resultados do uso do solo e planos ambientais raramente são monitorados ou avaliados após a implementação. Como resultado, os planejadores não podem saber se os planos atingem seus objetivos, como também não podem aprender a partir dos resultados de intervenções passadas para melhorar a prática de planejamento. Esta lacuna na avaliação é causada pela falta de métodos para avaliar os resultados dos planos e a dificuldade de se atribuir mudanças para atividades de planejamento Baer (1997) Oliveira;Pinho,(2010) Todes (2012) e Sharifi et al (2014) 
Dessa forma, uma importante questão relacionada com a problemática de degradação, relatada em alguns estudos, diz respeito a urbanização em áreas de preservação ambiental, o que pode ser observado e identificado nas cidades brasileiras através de vários tipos de conflitos entre uso e ocupação do solo que a legislação não esclarace. Assim, este trabalho tem como objetivo avaliar o desempenho do Plano Diretor de Desenvolvimento da Cidade de Pelotas/Rio Grande do Sul/Brasil em relação a Áreas de Preservação Ambiental.

\section{Materiais e métodos}

\subsection{A área de estudo}

As zonas de preservação ambiental da cidade de Pelotas, instituídas em lei de Plano Diretor de Desenvolvimento, configuram o caso em estudo (Figura 1). Ocupam uma área de 4216,5 há, onde habitavam em 201210.983 pessoas de acordo com um padrão espacial e socioeconômico heterogêneos. Nestas zonas existem três áreas distintas, localizadas a leste, a oeste e ao sul da cidade, na planície costeira sul do estado do Rio Grande do Sul/Brasil, sendo compostas por ambientes extremamente frágeis e ricos em biodiversidade. Fazem parte da franja destinada à expansão, compreendendo áreas de transição ou periurbanas. Nelas são identificadas várias ocupações em áreas públicas e inadequadas de acordo com Parfitt (2002) Parfitt (2016): banhados, dunas, borda de rio e áreas inundáveis por parcelamentos (loteamentos, ocupações informais, sítios de lazer).

No período pós-implementação do Plano Diretor houve expansão da área urbanizada em 520 hectares.

Nesse sentido, apesar da corrente deterioração observada nas áreas periurbanas da maioria das cidades brasileiras, o sistema de planejamento implementado não estabelece modelos de investigação dos conflitos decorrentes da produção do espaço urbano. Com o intuito de ampliar as formas de avaliação e controle de impactos ambientais, a estrutura teóricometodológica aqui apresentada - avaliação de desempenho do espaço produzido em Áreas Urbanas de Preservação Ambiental - está basicamente centrada em quatro obras: (Baer, 1997; Cunha, 1996; Bastos, 1996; e Coombes, 1993). 


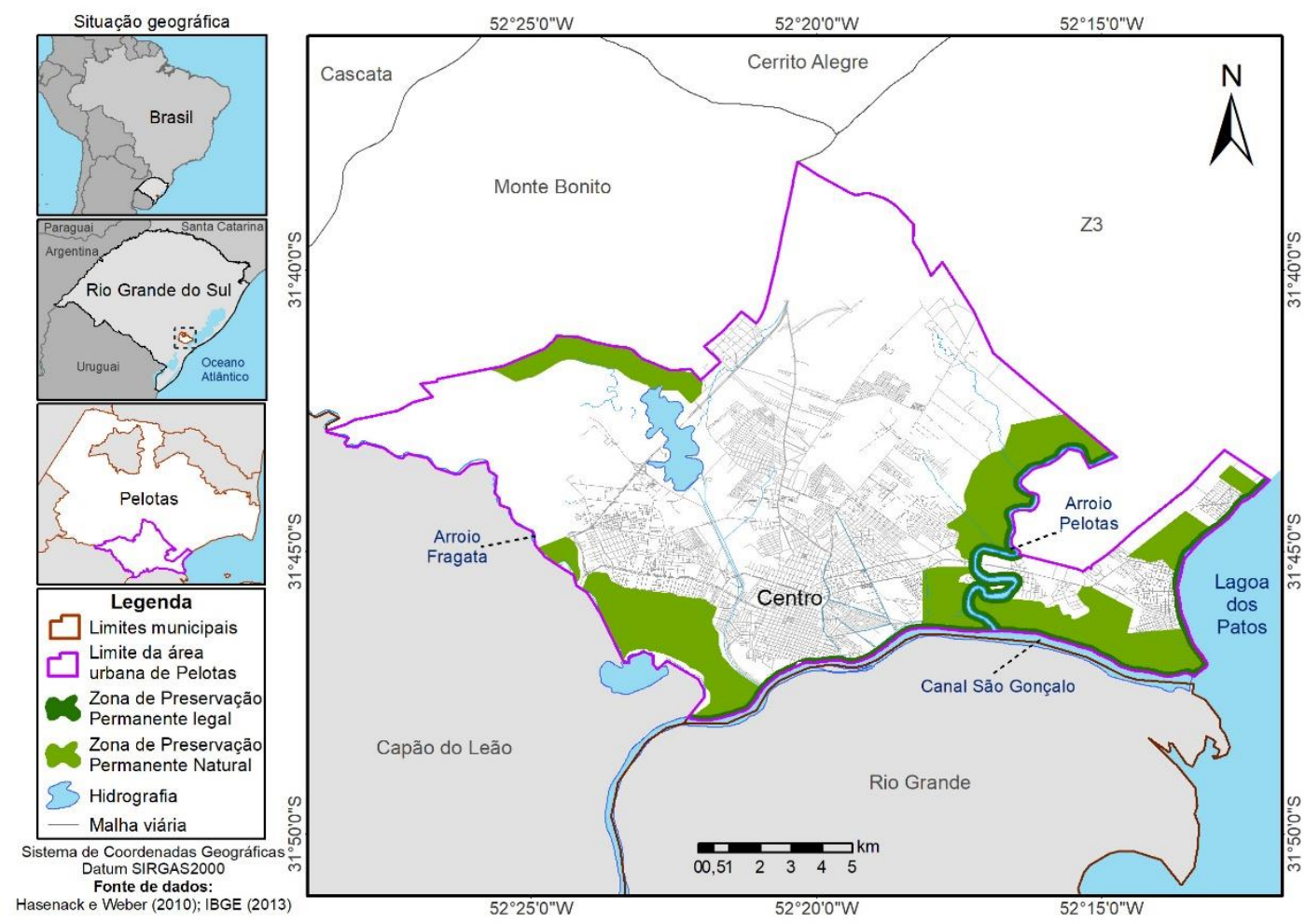

Figura 1- Zonas de Preservação Ambiental da cidade de Pelotas Fonte: Autores (2021)

Baer (1997) identifica tipos básicos de metodologias de avaliação de planos relacionados ao seu estágio de desenvolvimento, ou quando da implementação, durante ou após a sua elaboração.

Para o caso específico deste trabalho, será utilizada, a partir de Baer (1997), a avaliação pósimplementação, que consiste em comparar a performance proposta com os atuais resultados produzidos no espaço. Nesse processo, o plano é avaliado nos termos de seus resultados. 0 tipo de avaliação sugerida por ele é operacionalizada neste estudo nos seguintes termos: nas áreas estudadas o atual resultado é comparado à realidade anterior à implementação do plano, a partir de fotografias aéreas da cidade nos anos de 1980, 1995 e 2000.

O processo descrito por Baer (1997) equivale a estudo de impacto urbano, pois são avaliadas as condições ambientais na época posterior e anterior ao instrumento político implementado. Pode-se dizer que essa noção de impacto consiste em uma alteração ecossistêmica, sendo positivo (benéfico) e negativo (prejudicial), variando no espaço e no tempo dentro de uma realidade social. 
O segundo autor indicado, Cunha ( 1996) aborda o Estudo dos solos do município de Pelotas Esse estudo em conjunto com fotografias aéreas de 1995 serviu de base para o mapeamento das diferentes unidades Geomorfológicas do local, bem como a elaboração de mapa geomrfológico, base para avaliação da adequação a urbanização.

Já o trabalho de Bastos (1996) se refere ao Reconhecimento e caracterização geotécnica dos solos da Planície Costeira do Sul do RS. Esse estudo serviu para a identificação dos solos da área de estudo e sua adequação a urbanização.

Por fim, Coombes e Wong, (1994) tratam de maneira geral sobre estatística multivariada e sua utilização na análise de politica urbana e regional.

\subsection{Unidades de análise}

Para a avaliação do espaço produzido, as unidades de análise foram classificadas em dois tipos: Unidades Espaciais de Planejamento. 1) Unidades Geomorfológicas ou Ambientais 2) Unidades Espaciais de Desempenho.

\subsection{Unidades geomorfológicas (ambientais)}

Com a conceituação de sistema ecológico ou ecossistema como qualquer unidade abrangendo todos os organismos que funcionam em conjunto (comunidade biótica) numa dada área, interagindo no ambiente físico, de acordo com Odum (1953), o conceito pode ser relacionado com sociedade e natureza. Nessa perspectiva, uma fração da crosta terrestre pode ser qualificada de homogênea, pelo suporte e pela cobertura simultaneamente, mesmo existindo heterogeneidade de estrutura (soma de seres vivos diferentes, edificações e culturas).

Assim, propõe-se a análise funcional do ambiente humano a partir de uma interpretação dos

vários fatores levantados e rebatidos nas Unidades Geomorfológicas ou Ambientais (subdivisão do espaço com homogeneidade em relação aos diversos fatores analisados em uma extensão geográfica padrão), a fim de ser criado um modelo de diagnóstico de impactos de usos humanos em determinada região. 
Para este trabalho, a classificação dos compartimentos geomorfológicos segue Cunha (1996) e Parfitt (2002). Já a caracterização geotécnica teve por base Bastos (1996).

\subsection{Unidades Espaciais de Desempenho}

As unidades espaciais correspondem ao espaço urbano produzido definidas ou não pelo sistema de planejamento da cidade. Neste trabalho, as unidades de análise consistem em Unidades Espaciais de Planejamento (UEPs), zonas definidas a partir da lei de zoneamento da cidade, e Unidades Espaciais de Desempenho (UEDs), glebas-assentamentos nelas produzidos.

\subsection{Análise estatística multivariada}

Qualquer análise de dados referente a mais de duas variáveis é considerada uma análise multivariada. Para Coombes \& Wong (1993), essas técnicas se referem a um conjunto de procedimentos que torna possível a análise simultânea de medidas múltiplas para cada indivíduo, objeto ou fenômeno observado.

Šmilauer e Lepš (2014) evidenciam que a escolha do modelo estatístico mais adequado para descrever o comportamento de um grupo de variáveis depende da existência ou não de variáveis preditoras. Se elas não existirem, como no presente estudo, é possível resumi-las usando métodos de ordenação, entre os quais estão: análise de componentes principais (PCA), análise fatorial (FA) e análise de agrupamento (CA), entre outras.

De acordo com Hair et al. (2006), a Análise Fatorial, que inclui a análise de componentes principais e análise de eixos principais, é uma abordagem estatística que pode ser usada para analisar inter-relações entre um grande número de variáveis e explicar essas variáveis em termos de dimensões inerentes comuns (fatores). Esses autores, assim como alguns softwares estatísticos, como SPSS, consideram a Análise dos Componentes Principais (PCA) como um dos possíveis métodos a serem usados na Análise Fatorial (FA) para extração de fatores. Entretanto, conforme enfatizado por McGarigal, Cushman \& Stafford (2000), embora o objetivo destas técnicas seja o mesmo, o de resumir a estrutura de variância-covariância das variáveis estudadas gerando uma quantidade menor de componentes ou fatores 
responsáveis pelas alterações dos valores das variáveis originais, elas resumem a estrutura de covariância do conjunto de dados de maneira diferente. Na extração dos fatores, a PCA considera toda a variação existente nas variáveis, embora para cada uma delas exista uma parcela desta variação que lhe seja exclusiva. Já a FA separa a variação exclusiva da variação comum e ainda faz a suposição de que a intercorrelação entre as variáveis consideradas na análise é gerada por um número menor de variáveis latentes ou fatores. No presente estudo optou-se pela utilização de uma PCA com rotação varimax³ .

Outra técnica utilizada neste estudo foi a Análise de Agrupamento $\mathrm{CA},{ }^{4}$ que, de acordo com Hair et al. (2006) e McGarigal, Cushman \& Stafford (2000), tem o objetivo de classificar uma amostra de entidades em um pequeno número de grupos mutuamente excludentes, com base em suas similaridades, o que pode ser feito com a utilização de diferentes estratégias. Logo, esta técnica é capaz de determinar o desempenho conjunto das variáveis, bem como especificar a importância de cada uma delas, na presença das demais.

Em anos recentes, vários estudos têm utilizado a estatística multivariada em análise urbana e regional no Brasil. São exemplos os trabalhos de Jorge et al. (2010); Ribeiro et al (2014); Carvalho (2015).

\subsection{Avaliação de Desempenho}

Para avaliar o desempenho do espaço produzido em áreas de Preservação Ambiental, urbanas foram realizados os seguintes procedimentos básicos relacionados à regulamentação urbanística:

(1) identificar as zonas de interesse (UEPs) e sua regulamentação;

(2) identificar os assentamentos (UEDs) produzidos no período;

\footnotetext{
${ }^{3}$ Rotação dos eixos para identificar uma estrutura mais simples de dados. A varimax consiste em simplificar as colunas da matriz fatorial, fazendo com que algumas colunas tenham carga bem alta e outras próximas a zero. O método vai maximizar as cargas altas e minimizar as cargas baixas.

${ }^{4}$ Análise de agrupamento consiste em um conjunto de técnicas utilizadas para caracterizar objetos que podem ser variáveis ou unidades amostrais colocando-os em grupos similares. Essa análise pode ser feita em função das similaridades ou dissimilaridades.
} 
(3) identificar as variáveis na legislação (Plano Diretor de Desenvolvimento Urbano);

(4) classificar as variáveis identificadas no item anterior de acordo com indicadores ambientais e espaciais;

(5) formar um ranqueamento entre as UEDs com o objetivo de organizar os assentamentos em ordem decrescente, relacionando-os à regulamentação urbanística e à qualidade do espaço produzido;

(6) realizar a análise estatística com procedimentos multivariados, mais especificamente PCA e CA;

(7) identificar grupos de UEDs relativamente similares;

(8) mapear os resultados encontrados.

\section{Resultados}

A problemática no caso apresentado relaciona as Zonas de Preservação Ambiental com Unidades Espaciais de Planejamento (UEPs), conforme Figura 1, apresentando as Zonas de Preservação Ambiental instituídas no Plano Diretor, com a especificação das áreas estudadas e as glebas-assentamentos urbanos nelas produzidas com Unidades Espaciais de Desempenho (UED), como exemplo tem-se as figuras 2 e 3 e, ainda, paisagens com Unidades Geomorfológicas ou ambientais (UGs).

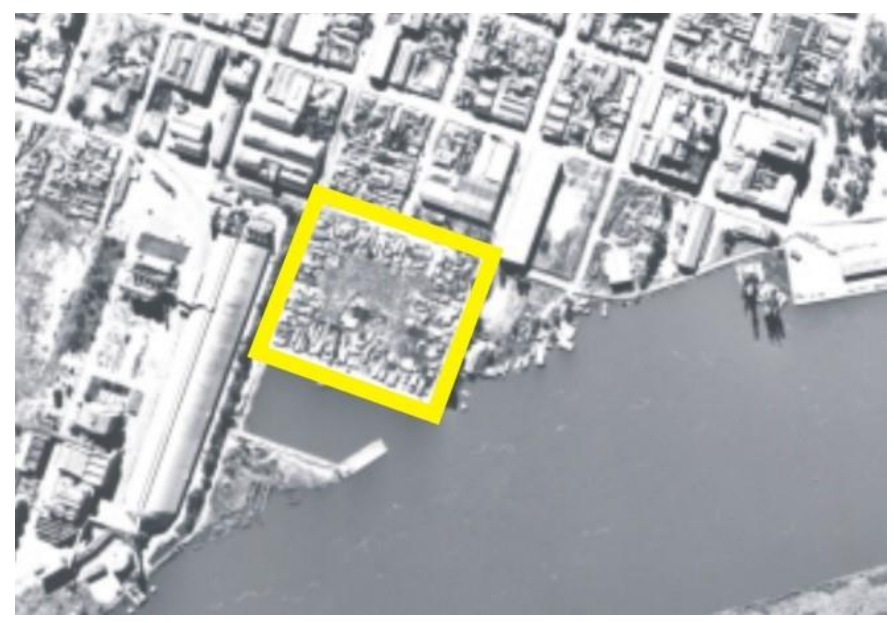

Figura 2 - UED 31 Doquinhas

Fonte: Fotografias aéreas Pelotas 1995 


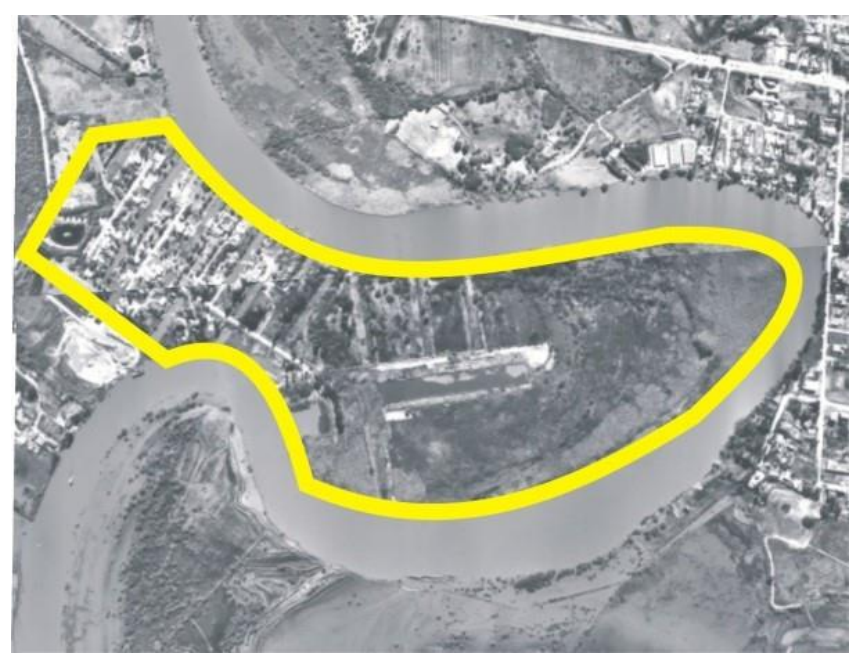

Figura 3 - UED Marina llha Verde

Fonte: Fotografias aéreas Pelotas 1995

Para facilitar a análise, as UEPs agregaram as diferentes zonas estabelecidas na área, instituídas pelo Plano Diretor, da seguinte forma:

1) UEP1 - compreende ZPPN leste (Zona de Preservação Paisagística Natural) e as zonas nela inseridas: ZEP, ZR4 e ZRM1, respectivamente Zona de Expansão Prioritária, Zona Residencial 4 (Santo Antônio/ Valverde) e Zona Residencial Mista 1, envolvendo o Barro Duro, área rural em período anterior a 1980;

2) UEP2 - compreende a Zona de Preservação Permanente Legal (ZPPL), faixas marginais a cursos de água nos termos do código florestal: Canal São Gonçalo, 100m; Arroio Pelotas, 50m e Lagoa dos Patos, $100 \mathrm{~m}$;

3) UEP3 - Zona de Preservação Paisagística Oeste (ZPPO), área de banhados possuindo somente uma ocupação.

Nesse processo, foram avaliadas 35 UEDs - assentamentos localizados nas três UEPs, conforme o Quadro 1.

Na visão da cidade como sistema produzido por diversos agentes e atores sociais e que está em constante transformação, é necessária a identificação de um conjunto de indicadores para o processo de planejamento que sirvam à análise morfológica de desempenho, no caso, Planos Diretores de Desenvolvimento Urbano, a fim de proporcionar o monitoramento da informação do espaço por parte dos gestores públicos. 
Esse procedimento permite detectar alterações espaciais ou temporais em parâmetros significativos, aumentar o conhecimento sobre as reações causa-efeito que explicam as variações e, inclusive, possibilitar a previsão de alterações futuras.

Neste trabalho, o conjunto de indicadores utilizados na análise (Avaliação de Desempenho) do espaço produzido nas Zonas de Preservação está relacionado com a legislação de controle do uso do solo da cidade de Pelotas que se encontra no Plano Diretor. Nela foram identificadas as variáveis relacionadas à problemática ambiental, de acordo com os objetivos aí expressos: no Capítulo I, quando da instituição das Zonas de Preservação Ambiental ZPPN leste e oeste, ZPPL (Zonas de Preservação Permanente Legal); no Capítulo II, quando se refere ao controle da produção do espaço nos processos de parcelamento do solo e leis complementares.

A partir do exposto, quatorze (14) variáveis foram identificadas, (referentes aos indicadores socioespaciais e socioambientais) e estão relacionadas aos objetivos da Lei do Plano Diretor de Desenvolvimento Urbano. Para cada uma delas, a UED recebe um escore compreendido entre 0 (zero) e 1 (um). 0 escore 0 corresponde à total inadequação, o escore 1 a total adequação, enquanto valores intermediários representam o atendimento parcial do requisito. A partir de então, é construído um banco de dados, utilizado em análises subsequentes: PCA e CA.

Paralelamente a esta análise, foi realizado um estudo para investigar a relação entre a pontuação atribuída as UEDs com dados socioeconômicos: valor da renda de seus moradores e valor do solo. Para avaliar a renda, foram consideradas 5 faixas salariais: 1) menos de dois salários mínimos; 2) de 2 até 4,99 salários mínimos; 3) de 5 até 9,99 salários mínimos; 4) 10 até 19,99; 5) 20 ou mais salários mínimos. Uma análise da variância encontra evidências de existência de diferença significativa entre as médias dos quatro grupos ( $F_{-}($calc.=12,974); $d f=3 ; d f=30 ; p$-value<0,001) e um teste de Schefée encontra evidências de que esta diferença ocorre em função do grupo que recebe menos de dois salários mínimos.

A relação entre a pontuação recebida pela UED e o valor do solo que lhe é atribuído também foi avaliada por meio do coeficiente de correlação de Pearson $(r=0,563$; t_(calc.)= ; df=33 ; pvalue $<0,01)$, sendo constatada a existência de uma correlação média, positiva e significativa, 
ou seja, foram encontradas evidências de que existe uma tendência de que UEDs com maiores pontuações tenham maiores valores de solo.

Assim, três tipos de informações referentes às condições, espaciais, ambientais e socioeconômicas dos assentamentos produzidos são relacionados à problemática da produção do espaço urbano nas áreas de preservação.

Os três indicadores utilizados na análise das UEPs e UEDs correspondem a: 1) Indicador Socioambiental, referente à produção do espaço no meio ambiente natural; 2) Indicador Socioespacial, relacionado com a estrutura do espaço produzido; a) Infraestrutura e Serviços urbanos b) Areas verdes Ocupação do Solo 3) Indicador Socioeconômico, próprio para a identificação dos padrões socioeconômicos, e encontram-se especificados nos Quadro 1 bem como as fontes de dados no Quadro 2.

Quadro1- Indicador Socioambiental; Indicador Socioespacial; Indicador Socioeconômico

\begin{tabular}{|c|c|c|}
\hline CATEGORIA & VARIÁVEL/ATRIBUTOS & SIGLA \\
\hline \multirow{5}{*}{$\begin{array}{l}\text { Indicador } \\
\text { Socio } \\
\text { Ambiental } \\
\text { Uso do solo }\end{array}$} & 1. Áreas Inundáveis (ocupação de áreas inundáveis) & Al \\
\hline & 2. Vegetação (ocupação de áreas de mata) & VG \\
\hline & 3. Poluição do solo (ocupação de áreas aterradas com material nocivo à saúde) & PS \\
\hline & 4. Solos (ocupacão de área com risco geotécnico) & ARG \\
\hline & $\begin{array}{l}\text { 5.Condições sanitárias (ocupação de áreas de risco inadequadas em relação às } \\
\text { condições sanitárias, poluição sonora, atmosférica ,da água ou outra) }\end{array}$ & CS \\
\hline \multirow{6}{*}{$\begin{array}{c}\text { Indicador } \\
\text { Socioespacial } \\
\text { (a) }\end{array}$} & 1. Sistema de abastecimento de água. & SAA \\
\hline & 2. Sistema de coleta de esgotos & SCE \\
\hline & 3. Sistema de energia elétrica domiciliar & SEE \\
\hline & 4. Sistema de coleta de lixo domiciliar & $\mathrm{SCL}$ \\
\hline & 5. Sistema de iluminação pública & SIP \\
\hline & 6. Sistema de drenagem & SD \\
\hline \multirow{3}{*}{$\begin{array}{c}\text { Indicador } \\
\text { Socioespacial } \\
\text { (b) }\end{array}$} & 1 Áreas verdes & AV \\
\hline & 2 Arborização de vias & AVP \\
\hline & 3.Ocupação de áreas públicas & OAP \\
\hline \multirow{6}{*}{$\begin{array}{l}\text { Indicador } \\
\text { Socio } \\
\text { econômico }\end{array}$} & 1. Renda-nível classificação salarial & NR \\
\hline & 2. Gradientes valor do solo & VS \\
\hline & 3. Número de habitantes UEP/UED & POP \\
\hline & 4 Tipo de assentamento & SF \\
\hline & -Regular & \\
\hline & -Irregular (espontâneo; clandestino) & \\
\hline
\end{tabular}

Fonte: Autores (2021) 
Quadro 2 - Variável/ Atributos e Fonte de dados

\begin{tabular}{|c|c|c|}
\hline VARIÁVEL /ATRIBUTOS & FONTE /DADOS & SIGLA \\
\hline $\begin{array}{l}\text { 1. Áreas Inundáveis (ocupação de } \\
\text { áreas inundáveis) }\end{array}$ & Mapa áreas risco inundação & $\mathrm{Al}$ \\
\hline $\begin{array}{l}\text { 2. Vegetação (ocupação de áreas de } \\
\text { mata) }\end{array}$ & Fotografias Aereas ou Goole Earth & VG \\
\hline $\begin{array}{l}\text { 3. Poluição do solo (ocupação de áreas } \\
\text { aterradas com material nocivo à } \\
\text { saúde) }\end{array}$ & Prefeitura Municipal & PS \\
\hline $\begin{array}{l}\text { 4. Solos (ocupação de área com risco } \\
\text { geotécnico) }\end{array}$ & Mapa Geotécnico & ARG \\
\hline 1. Sistema de abastecimento de água. & Prefeitura Municipal & SAA \\
\hline 2. Sistema de coleta de esgotos & Prefeitura Municipal & SCE \\
\hline $\begin{array}{l}\text { 3. Sistema de energia elétrica } \\
\text { domiciliar }\end{array}$ & CEEE empreesa energia & SEE \\
\hline 4. Sistema de coleta de lixo domiciliar & Prefeitura Municipal & $\mathrm{SCL}$ \\
\hline 5. Sistema de iluminação pública & Prefeitura Municipal & SIP \\
\hline 6. Sistema de drenagem & Prefeitura Municipal & SD \\
\hline 1 Áreas verdes & Fotografias Aereas ou Goole Earth & $\mathrm{AV}$ \\
\hline 2 Arborização de vias & Fotografias Aereas ou Goole Earth & AVP \\
\hline 3.Ocupação de áreas públicas & Prefeitura Municipal & OAP \\
\hline 1. Renda-nível classificação salarial & $\begin{array}{l}\text { Instituto Brasileiro de Gografia e } \\
\text { Estatística IBGE }\end{array}$ & NR \\
\hline 2. Gradientes valor do solo & Prefeitura Municipal & VS \\
\hline 3. Número de habitantes UEP/UED & $\begin{array}{l}\text { Instituto Brasileiro de Gografia e } \\
\text { Estatística IBGE }\end{array}$ & POP \\
\hline $\begin{array}{l}4 \text { Tipo de assentamento } \\
\text {-Regular } \\
\text {-Irregular (espontâneo; clandestino) }\end{array}$ & Prefeitura Municipal & SF \\
\hline
\end{tabular}

Fonte: Autores (2021) 


\section{Análise dos resultados}

\subsection{Formação do ranking}

O objetivo desse procedimento consistiu em organizar um ranking de assentamentos em ordem decrescente, relacionando a regulamentação urbanística (lei do Plano Diretor de Desenvolvimento Urbano) à qualidade ambiental do espaço produzido, o que foi feito a partir do somatório dos pontos recebidos pelas UEDs. Aos critérios 1, 3 e 5 referentes a áreas inundáveis, poluição do solo e condições sanitárias foi atribuído o peso 2 em função de estarem relacionados à área de risco Quadro 3.

Quadro 3: Ranking das UEDs de acordo com a pontuação recebida

\begin{tabular}{|c|c|c|c|}
\hline Código UED & Nome do Assentamento & Pontuação & Posição \\
\hline 2 & Colina Verde & 17,00 & 1 \\
\hline 14 & Vila Assumpção 1 & 17,00 & 1 \\
\hline 1 & Vila Mariana & 16,00 & 3 \\
\hline 3 & Las Acácias & 15,50 & 4 \\
\hline 12 & Vela Bela & 15,50 & 4 \\
\hline 11 & São Conrado & 14,00 & 6 \\
\hline 19 & Vila Judite & 14,00 & 6 \\
\hline 16 & Parcelamento dos Oliveira & 13,50 & 8 \\
\hline 9 & Barro Duro & 13,25 & 9 \\
\hline 8 & Marina Ilha Verde & 13,00 & 10 \\
\hline 13 & Vila Assunção II & 13,00 & 10 \\
\hline 26 & Marina Ilha Verde & 13,00 & 10 \\
\hline 10 & Santo Antônio & 12,50 & 12 \\
\hline 18 & Costa Doce & 12,50 & 12 \\
\hline 17 & Carmelo & 12,12 & 14 \\
\hline 15 & Colina Verde II & 12,00 & 15 \\
\hline 5 & Golden Village & 11,30 & 16 \\
\hline 4 & Ana Estela & 11,00 & 17 \\
\hline 22 & PraçaAratiba & 10,50 & 18 \\
\hline 24 & Margem do Canal & 10,50 & 18 \\
\hline 6 & Recanto de Portugal & 9,90 & 20 \\
\hline 25 & Recanto de Portugal & 9,90 & 20 \\
\hline
\end{tabular}




\begin{tabular}{lcll}
\hline 21 & Quarteirão da CEEE & 9,50 & 22 \\
\hline 34 & Vila da Palha & 9,50 & 22 \\
\hline 7 & Pontal da Barra & 9,30 & 24 \\
\hline 33 & Meneguetti & 9,00 & 25 \\
\hline 32 & Balsa & 8,16 & 26 \\
\hline 23 & Praça do Laranjal & 8,05 & 27 \\
\hline 20 & Novo Valverde & 7,50 & 28 \\
\hline 31 & Doquinhas & 7,49 & 29 \\
\hline 29 & Rua Silveira Calheca & 6,73 & 30 \\
\hline 28 & Núcleo de Psc. P. da Barra & 6,00 & 31 \\
\hline 30 & Clube do Remo & 5,55 & 32 \\
\hline 27 & Núcleo de Psc do trapiche & 5,00 & 33 \\
\hline 35 & Ponte do Rio Grande & 2,19 & 34 \\
\hline
\end{tabular}

Fonte: Autores (2021)

De acordo com esta pontuação, as duas melhores UEDs receberam a totalidade dos pontos e são Colina Verde e Vila Assumpção I, seguidas de Vila Mariana. A pior UED foi a Ponte do Rio Grande.

Os dois pertencem à UEP1 ocupam áreas altas na unidade geomorfológica correspondente às lombadas costeiras arenosas, possuem toda a infraestrutura, áreas verdes e arborização de vias. São ocupados por população com renda superior a 10 salários mínimos, possuindo situação fundiária regular.

O assentamento Ponte do Rio Grande, com menor pontuação, consiste em um assentamento clandestino ${ }^{5}$, o único localizado na UEP3, ZPPN oeste. Ao contrário dos que receberam maior pontuação e seguem todos os requisitos estabelecidos na lei, encontra-se em área de risco de inundação, poluição do solo e da água, bem como de acidentes, (por estar localizado ao lado de uma rodovia). Praticamente não possui nenhum tipo de infraestrutura e áreas verdes, à exceção de ligações de água e de energia elétrica em parte das residências.

\footnotetext{
${ }^{5}$ Assentamentos Clandestinos: Nesses assentamentos as construções são realizadas em terrenos invadidos e sem regularização fundiária e suas edificações são inadequadas, feitas com materiais precários, em locais sem qualquer apoio estatal, sem energia elétrica, abastecimento de água, esgoto, etc.
} 
Além deste, os três anteriores, que ocupam a posição 33, posição 32 e posição 31 no ranking estão ocupados por assentamentos informais e clandestinos, cujos moradores têm renda entre zero a dois salários mínimos, a exceção do loteamento Pontal da Barra.

Esta avaliação, entretanto, não considera a concordância ou discordância entre os diversos critérios considerados, razão pela qual se optou por complementar esta análise com métodos estatísticos multivariados por permitirem a análise simultânea de várias variáveis, colocando em evidência suas semelhanças e diferenças.

No desenvolvimento da PCA, primeiramente foram encontrados os autovalores, o que permitiu identificar seis fatores definidos como uma combinação linear entre as variáveis consideradas no estudo, com resultado superior a um para o autovalor. Os dois primeiros, entretanto, conseguem explicar $44 \%$ da variabilidade das variáveis consideradas na avaliação das UEDs. A Figura 4 apresenta estes dois fatores, evidenciando que é possível identificar UEDs similares e dissimilares.

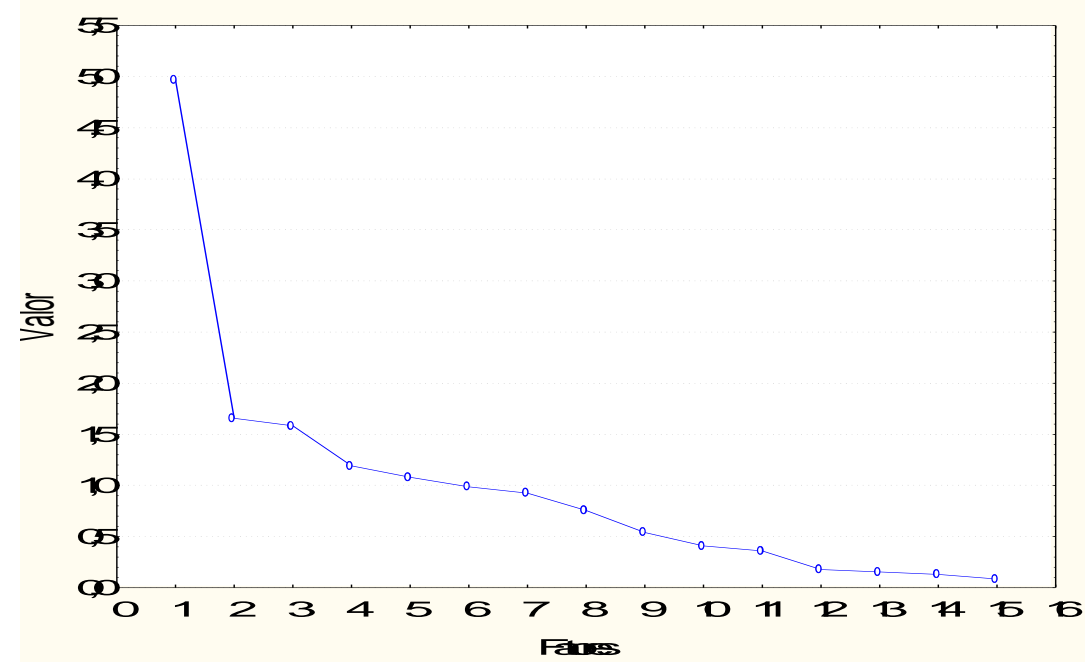

Figura 4 - Distribuição dos UEDs de acordo com os fatores selecionados Fonte: Autores (2021)

Já no gráfico da figura 5 são identificados três grupos, sendo o primeiro subdividido em três, enquanto o segundo, em dois e o terceiro em um. Quatro assentamentos, entretanto, não são alocados em nenhum dos grupos. 


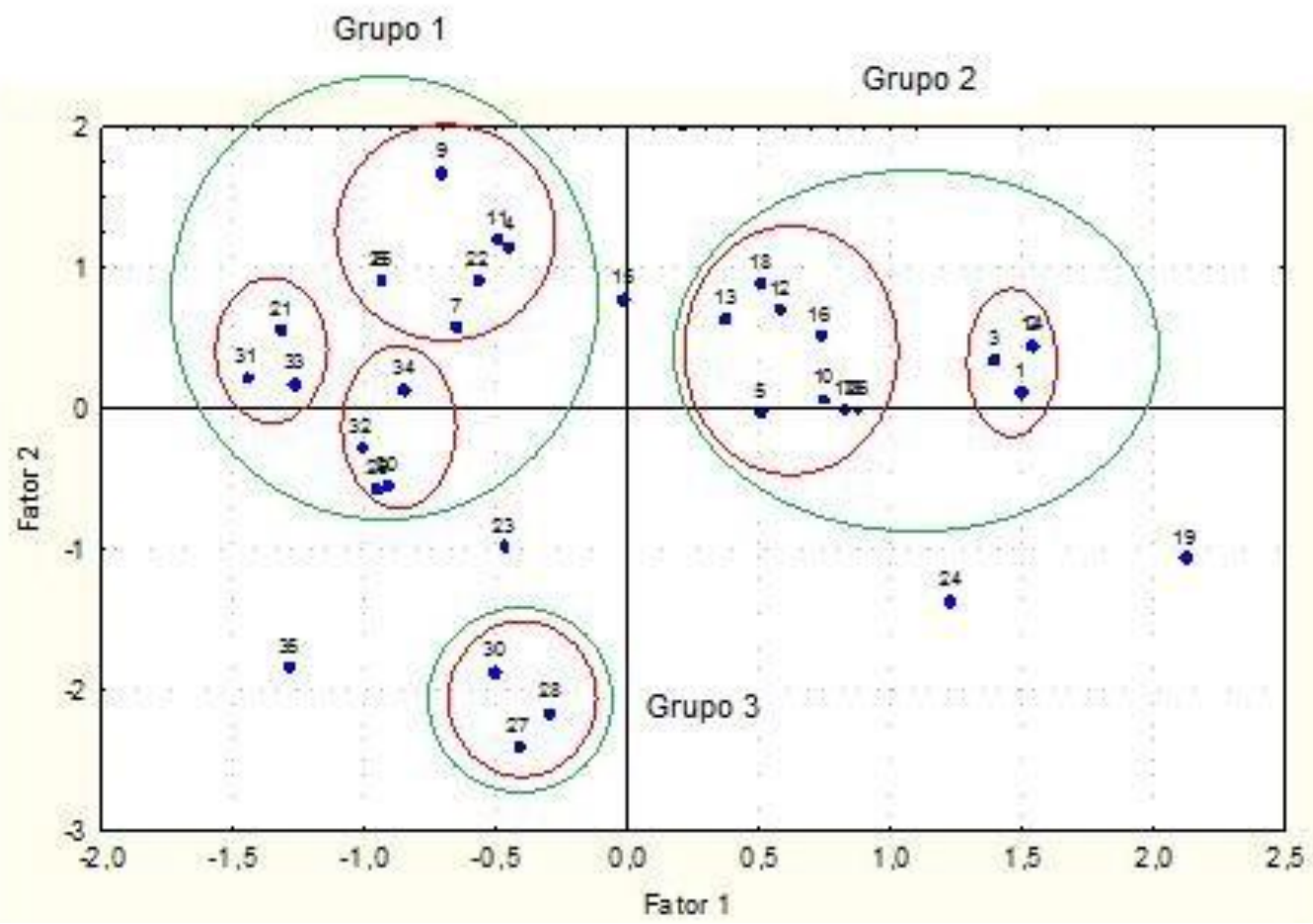

Figura 5 - Distribuição dos UEDs de acordo com grupos selecionados Fonte: Autores (2021)

Para a identificação das diferentes UEDs em grupos relativamente similares, esta análise foi complementada por uma outra técnica: CA. Em sua aplicação foi utilizada a distância euclidiana para quantificar similaridades ou dissimilaridades; o método de ligação completa ou vizinho mais longe para a definição formal dos grupos e a árvore hierárquica como critério para identificar a composição dos seis grupos. Estes procedimentos possibilitaram a construção do Dendograma apresentado na Figura 6.

A análise do gráfico permitiu identificar a composição dos grupos organizados em ordem decrescente de acordo com a qualidade do espaço produzido. Considerando uma distância máxima de 1,5 para a formação de um grupo, são identificados seis grupos:

- Grupo 1: assentamentos 6, 7, 21, 25, 31, 33, 34;

- Grupo 2: assentamentos 4, 9, 11, 15, 22;

- Grupo 3: assentamentos 27, 28, 30, 35;

- Grupo 4: assentamentos 20, 23, 29, 32;

- Grupo 5: assentamentos 19, 24;

- Grupo 6: assentamentos 1, 2, 3, 5, 8, 10, 12, 13, 14, 16, 17, 18, 26. 


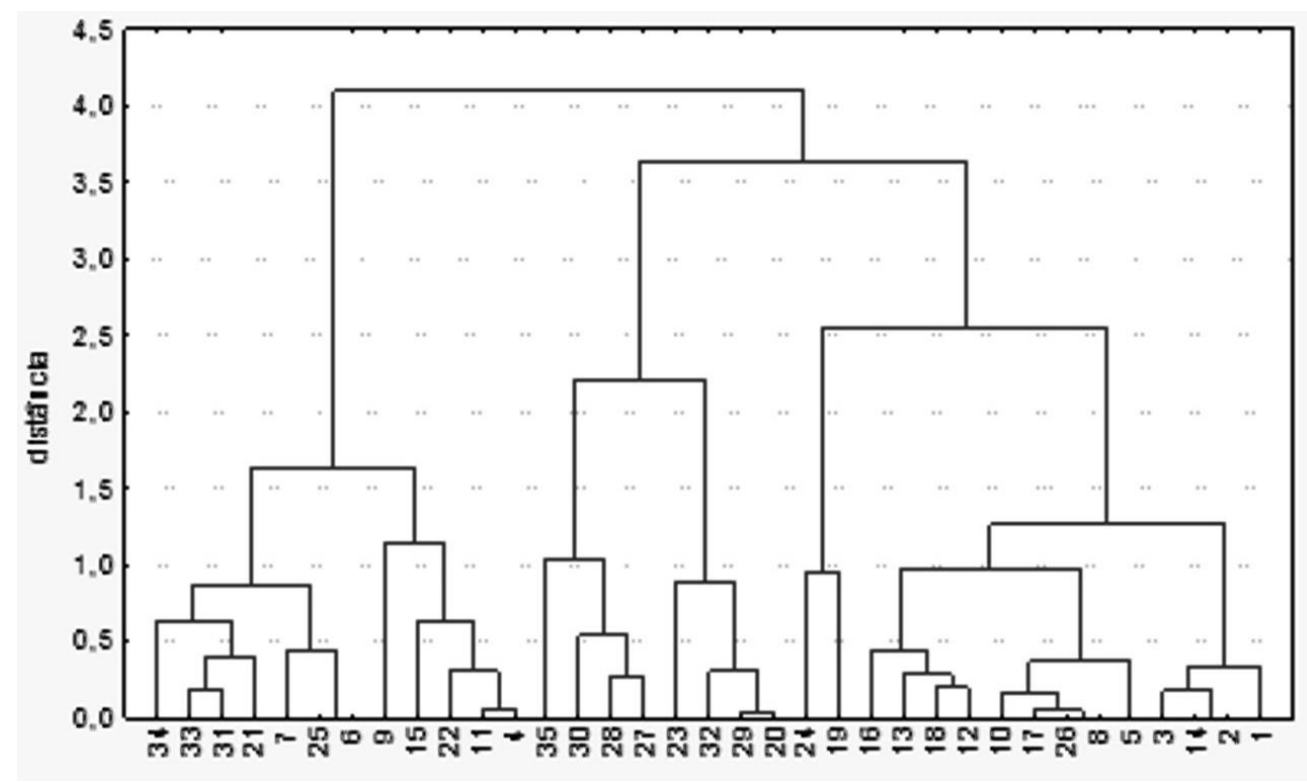

Figura 6 - Dendograma

Fonte: Autores (2021)

Para a composição final dos grupos bem como seu ordenamento, foram levados em consideração os resultados obtidos pela PCA e pela CA, assim como as médias das pontuações recebidas no ranking de assentamentos e características analisadas.

\section{Grupo 1}

Subgrupo 1a - Assentamentos com moradores que possuem renda superior a 20 salários mínimos; ${ }^{6}$ formado por loteamentos ou sítios de lazer localizados em áreas altas, com toda a infraestrutura, áreas verdes e arborização de vias; inadequação quanto a solos na UED1 e UED3 (localizados em áreas com indicativo à preservação ambiental, dunas) ${ }^{7}$. Pontuação média no ranking: 16.37.

Subgrupo 1b - Assentamentos com moradores que possuem renda superior a 20 salários mínimos; formado por loteamentos, condomínios ou desmembramento regulares, irregulares e clandestinos; presença de infraestrutura, arborização de vias e áreas verdes, a maior parte amorfas; localizados em áreas altas ou margem de corpos de água; ocupação em

\footnotetext{
${ }^{6}$ Em 2021, o salário mínimo no Brasil está valendo 192 dólares (1.100 reais), o menor valor da década.

${ }^{7}$ Villaça (2001) comenta que "as camadas de alta renda, não tem preferência sobre sítios altos ou baixos, mas sim os melhores, segundo os valores sociais e as condições de segurança, salubridade e beleza no contexto de cada situação histórica, atendido o requisito da acessibilidade".
} 
áreas de mata e dunas; inadequação quanto a solos na UED 13. Pontuação média no ranking: 12.9.

Grupo 2 - Assentamentos com moradores que possuem renda entre 10 e 20 salários mínimos; formado por sítios de lazer clandestinos; ausência de recolhimento de lixo e iluminação pública; presença de arborização de vias. Pontuação média no ranking: 12,25.

Grupo 3 - Assentamentos com moradores que possuem renda entre 5 e 10 salários mínimos; formado por loteamento irregulares e clandestinos; localização em área de mata nativa; presença parcial de infraestrutura UED9 e UED22; sistema de abastecimento de água por poço artesiano; ausência de áreas verdes e arborização de vias. Pontuação média do ranking: 12.15 .

Grupo 4 - Assentamentos com moradores que possuem renda entre 0 e 2 salários mínimos; formado por loteamentos e assentamentos espontâneos, clandestinos e irregulares; localizados em áreas propícias à inundação; presença parcial de infraestrutura na UED6; abastecimento de água por poço artesiano; deficiência no sistema e drenagem; ausência de arborização de vias e deficiência de áreas verdes. Pontuação média no ranking: 9.22.

Grupo 5 - Assentamentos com moradores que possuem renda entre 0 e 3 salários mínimos; formado assentamentos clandestinos espontâneos; localizados em áreas públicas, próximas ao centro ou nas imediações do Pontal da Barra; infraestrutura precária, ausência de áreas verdes e arborização de vias; deficiência de drenagem; área propícia à inundação. Pontuação média no ranking: 7.61 .

Grupo 6- Assentamentos com moradores que possuem renda entre 0 e 1 salário mínimo; formado assentamentos clandestinos espontâneos; habitações de madeira autoconstruídas, localizados em áreas públicas com risco de inundação na margem do canal São Gonçalo; próximas ao centro e Pontal da Barra; infraestrutura precária, ausência de arborização de vias e áreas verdes. Pontuação média no ranking: 4.68.

Os resultados obtidos foram espacializados e encontram-se na Figura 7. 


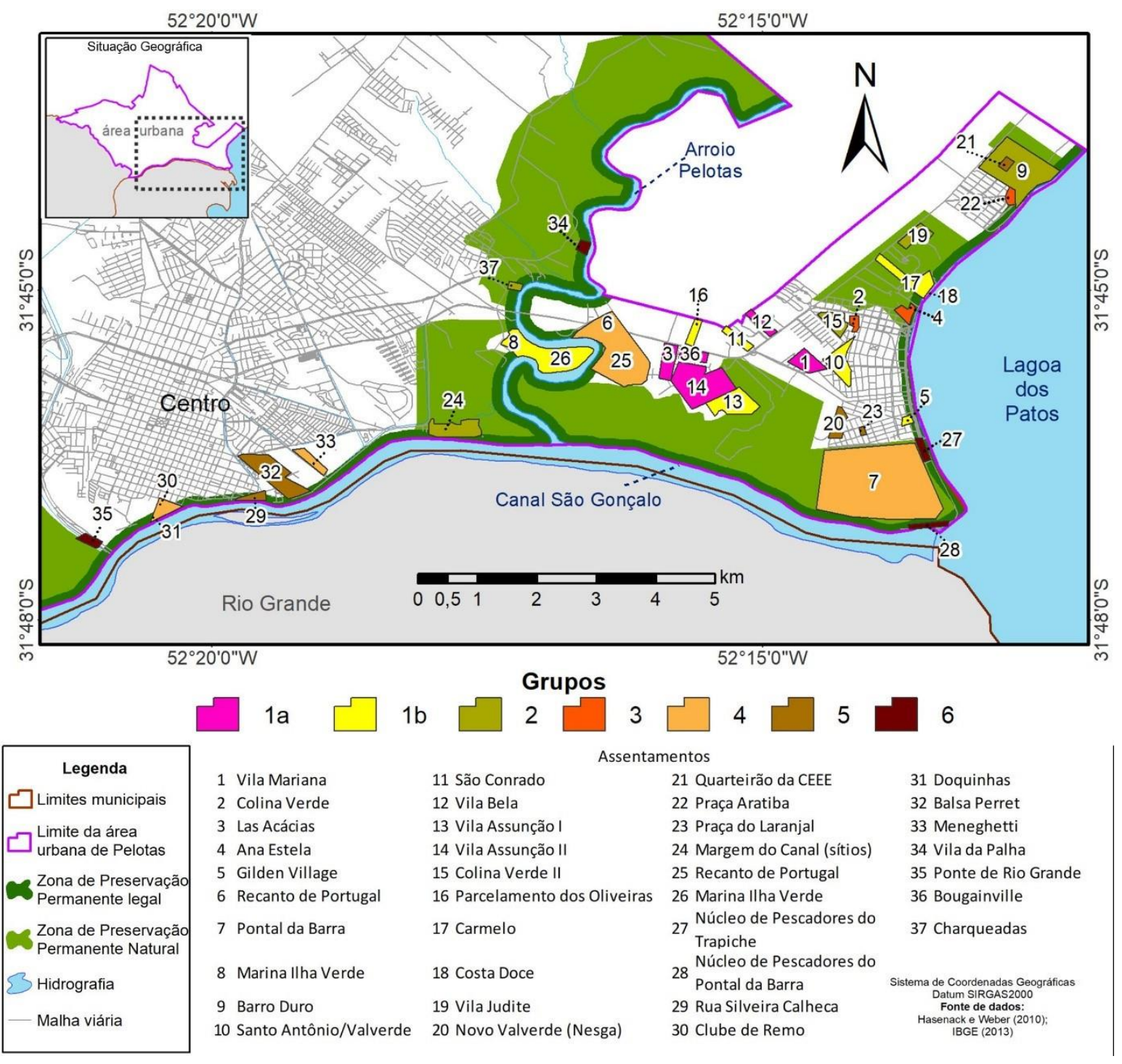

Figura 7- Distribuição dos assentamentos por grupos

Fonte: Autores (2021)

A análise geral de dados referente à composição identifica a presença de três conjuntos, sendo o primeiro formado pelos grupos de maior renda, assentamentos regulares, irregulares ou clandestinos e dotados de infraestrutura, alguns localizados em áreas inadequadas do ponto de vista ambiental, causando importantes impactos no meio ambiente, principalmente com a ocupação de áreas de mata e dunas. Correspondem predominantemente aos assentamentos dos grupos 1 e 2 .

O segundo constitui uma situação intermediária, composta por assentamentos irregulares ou clandestinos, apresentando algumas deficiências vinculadas à infraestrutura. Em relação ao aspecto ambiental, os maiores impactos consistem na ocupação de áreas de mata nativa, 
dunas, banhado e inadequadas do ponto de vista de drenagem. Correspondem a predominantemente os assentamentos dos grupos 3 e 4 . O terceiro, se refere a assentamentos espontâneos clandestinos, localizados em áreas públicas, com infraestrutura precária e de risco, sendo constituído pelos assentamentos dos grupos 5 e 6 .

Dessa forma, cabe salientar no caso estudado que a produção do espaço urbano consiste em uma manifestação espacial dos conflitos de interesses dos diversos agentes, presentes nesse processo, associado ao desenvolvimento capitalista.

A produção do espaço urbano é na verdade a produção de localizações. As diferentes localizações apresentam diferentes valores associados ao valor dos elementos urbanos existentes, incluindo a acessibilidade aos bens e serviços. A diferença entre os valores das localizações explica as diferentes configurações e as diferenças de valor das áreas urbanas (Villaça, 2001). Assim, os estratos de maior renda beneficiam-se desse processo diferentemente dos mais pobres, que permanecem à margem, por não disporem de recursos econômicos.

É importante destacar também, a questão da ilegalidade da propriedade do solo urbano. Maricato (2013) afirma que ela atinge mais de 50\% nas grandes cidades brasileiras considerando as legislações de uso e ocupação do solo, zoneamento, parcelamento do solo e edificação. A ilegalidadede acordo com a autora porém é uma das marcas da cidade brasileira, para além das metrópoles Essa ilegalidade acontece devido ao baixo rendimento de grande parte da população, como a pouca oferta de terras no mercado imobiliário formal. A população de menor poder aquisitivo tende a ocupar áreas desvalorizadas pelo mercado imobiliário, como a periferia urbana precária em serviços e regiões ambientalmente frágeis encostas, fundos de vale, áreas sujeitas a inundações, áreas de proteção ambiental (Maricato, 2013). Aqui, todavia, verifica-se que há também indivíduos com renda mais elevada em situação ilegal, o que resulta da qualidade ambiental e das características da localização.

Para concluir, tanto os grupos de maior, quanto os de menor renda causam impactos no meio ambiente a partir da produção do espaço nas áreas estudadas. As populações de assentamentos precários apresentam três aspectos: insalubridade, vulnerabilidade social e situação de risco, enquanto que as com maior renda buscam os atrativos do sítio. 
Assim, tem-se o desafio de lidar com as resultantes do processo de produção do espaço urbano e fazer com que a prática de planejamento contribua para atenuar a exclusão social e a degradação ambiental.

Nesse sentido, com a premissa de que Planos Diretores têm pouco poder de orientar o desenvolvimento urbano, Todes (2012) e Ayyoob (2014), lembram a importância da utilização de sistemas de monitoramento, como o apresentado, para o acompanhamento de Planos Diretores e a gestão de áreas urbanas, que incluam informação rigorosa e recortes espaciais precisos, a par de práticas participativas.

\section{Considerações finais}

Partindo do princípio de que o processo de urbanização identificado nas cidades brasileiras se desenvolve com desrespeito com o que é proposto pelos Planos Diretores de Desenvolvimento e que existe uma oportunidade de integrar o desenvolvimento urbano através de novos instrumentos de gestão no sistema de planejamento, esta pesquisa desenvolveu uma estrutura de análise da produção do espaço para zonas de Preservação Ambiental Urbanas.

Mostram-se importantes os diferentes tipos de procedimentos utilizados para a avaliação do espaço produzido, como a classificação hierárquica e a formação de grupos de assentamentos com base em características similares relacionadas às variáveis de análise, na medida em que podem vir a apoiar processos de decisão, designadamente no licenciamento, através de sistemas de controle periódico (monitoramento) do meio ambiente urbano.

Embora reconhecendo que a metodologia utilizada apresenta algumas limitações por depender do conhecimento do analista frente ao problema proposto, assim como das dificuldades na obtenção de dados, utilizar a avaliação de desempenho associada à regulamentação em vigor permitiu demonstrar a sua aplicabilidade para o caso específico.

Considerações acerca dos processos naturais entre a cidade e o ambiente; a busca de novas estratégias, gestão das rendas fundiárias (públicas e privadas) pelo poder público através da análise de suas formas de geração e apropriação e a utilização de instrumentos de planejamento e gestão mais dinâmicos se fazem necessários. A integração com um Sistema 
de Informações Geográficas, a agregação de um número maior de variáveis e a análise de dados por indicador ou por variável seriam algumas das possibilidades de novas abordagens.

\section{Referências}

Akbulut, A.; Ozcevik, O.; Carton, L. (2018) "Evaluating suitability of a GIS-AHP combined method for sustainable urban and environmental planning in Beykoz district, Istanbul", Int. J. Sus. Dev. Plann. 13(8), p. 1103-1115. https://doi.org/10.2495/SDP-V13-N8-1103-1115

Ali L, Nawaz A, Iqbal S, Basheer MA, Hameed J, Albasher G, Adnan S, Shah R, Bai Y. . (2021) Dynamics of transit orienteddevelopment, role of greenhouse gases and urban environment: A study for management and policy. Sustainability. 13:2536. doi: 10.3390/SU13052536

Baer, W. (1997) General plan evaluation criteria: An approach, to making better plans. Chicago: Journal of American Planning Association, Chicago, v. 63, n.3, p.329-345.

Bastos, C. (1996) FAPERGS-FUNDAÇÃO DE APOIO A PESQUISA DO RIO GRANDE DO SUL Reconhecimento e características geotécnicas dos solos da planície costeira sul do RS: Relatório de Pesquisa. Porto Alegre, 96p.

Carvalho, G. A. (2015) Contribuições metodológicas ao Planejamento Urbano: comparação de métodos de análise multivariada e modelos de mudança da paisagem urbana - aplicações na Regional Pampulha - Belo Horizonte, Minas Gerais - Brasil. Tese de Doutorado, Instituto de Geociências, UFMG, 155 p.

Calthorpe, Peter, (1996)'The Pedestrian Pocket', in Richard LeGates and Frederic Stout (eds) The City Reader, London: Routledge,pp. 468-74.

Coombes, M.; Wong, C. (1994) Methodological steps in the development of multivariate indexes for urban and regional policy analysis. Environment and Planning A, London, U.K.,v.26,p.1297-1316.

Cunha, N. (1996) Estudo dos solos do município de Pelotas. Documentos, EMBRAPA, Pelotas: CPACT.67p.

Duany, A., Speck, J., Lydon, M. (2010) The Smart Growth Manual. New York: McGraw-Hill.

Gottdiener, M. (1997) A produção social do espaço urbano. São Paulo: Edusp, 249p.

Guyadeen, D.; Seasons, M. (2018) Evaluation theory and practice: Comparing program evaluation and evaluation in planning. Journal of Planning Education and Research, Austin, n. 38, 98-110 https://doi.org/10.1177/0739456X16675930

Hair, J., Black, W., Babin, B., Anderson, R.; Tatham, R. (2006) Multivariate Data Analysis Sixth Edition, Prentice Hall, Upper Saddle River, New Jersey.

Harvey, D., (1980). A Justiça Social e a Cidade. São Paulo: Hucitec,1980.257p.

Hersperger, A. M., Oliveira, E., Pagliarin, S., Palka, G., Verburg, P., Bolliger, J., \& Grădinaru, S. Urban land-use change: The role of strategic spatial planning, Global Environmental Change, UK, v. 51, p. 32-42, 2018. doi: 10.1016/j.gloenvcha.2018.05.001

Jorge, M.A.; Frei,F.; Sales,J.M.S.; Lima,B.M. (2010) Cálculo e implementação do índice de desenvolvimento da gestão municipal (IDGM) do município de Itabaiana/SE. Planejamento e Políticas Públicas. Rio de Janeiro, v.34, p.9-34.

Ullah K.M. \& Mansourian A., (2015) Evaluation of land suitability for urban land-use planning: case study Dhaka City. Transactions in GIS, 20(1), pp. 20-37, 2015. https://doi.org/10.1111/tgis.12137

MARICATO, E. Cidades no Brasil: neo desenvolvimentismo ou crescimento periférico predatório? In: Revista Política Social e Desenvolvimento. n.1, Campinas: Plataforma Política Social, 2013.

MARTINE, G. (2011) Preparing for sustainable urban growth in developing areas. In: UNDESA (Ed.) Population distribution, urbanization, internal migration and development: An international perspective. New York, NY: United Nations, p. 6-30. 
McGARIGAL, K., CUSHMAN, S.; STAFFORD, S. (2000) Multivariate statistics for wildlife and ecology research. New York: Springer-Verlag.

Montgomery, B.; Dragićević, S. (2016) Comparison of GIS-based logic scoring of preference and multicriteria evaluation methods: Urban landuse suitability. Geographical Analysis, Columbus,48(4), p. 427-447. https://doi.org/10.1111/gean.12103

Odum.E. (1953) Fundamentals of Ecology. Philadelphia: W.B. Saunders Company,1953.632p.

Oliveira, V.; Pinho, P. Evaluation in urban planning: advances and prospects. (2010) Journal of Planning Literature, Londres, v.24, n.4, p. 343-361.

Parfitt, C. M. (2002)Impacto urbano em áreas de interesse e proteção ambiental: Produção do espaço em zonas de preservação ambiental da cidade de Pelotas/RS, na lei 2565/80. 2002. Dissertação (Mestrado- Pós Graduação em Planejamento Urbano Regional) - Faculdade de Arquitetura, Universidade Federal do Rio Grande do Sul, Porto Alegre.

Parfitt CM.(2016) Áreas de preservação do ambiente natural urbano, segregação e impacto nas paisagens e na biodiversidade: estudo de caso de Pelotas RS. Raega. 37:07-36.

PELOTAS, Lei n.2565 de 1980, II Plano Diretor de Desenvolvimento Urbano. Disponível em:<http://www2.camarapel.rs.gov.br.>Acesso em: 14 de maio de 2020.

Pupphachai,U.; Zuidema,C. (2017) Sustainability indicators: A tool to generate learning and adaptation in sustainable urban development. Ecological Indicators, Amsterdam, v.72, p. 784-793. https://doi.org/10.1016/j.ecolind.2016.09.016

Raddad, S. (2016) Integrated a GIS and multi criteria evaluation approach for suitability analysis of urban expansion in southeastern Jerusalem region-Palestine. American Journal of Geographic Information System, 5(1), 24-31.

Sarkodie SA, Owusu PA, Leirvik T (2020) Global effect of urban sprawl, industrialization, trade and economic development on carbon dioxide emissions. Environ Res Lett 15(3):34049

Sharifi, A., Chibab, Y., Okamotoc, K., Yokoyamac, S., Murayamad, A., (2014) Can master planning control an regulate urban growth in Vientiane, Laos? Landscape and Urban Planning, Amsterdam, v.131, p.1-13. http://dx.doi.org/10.1016/j.landurbplan.2014.07.014

Shen, L., Shuai, C., Jiao, L., Tan, Y., Song, X., 2017. Dynamic sustainability performance during urbanization process between BRICS countries. Habitat Int. 60, 19-33. https://doi.org/10.1016/j.habitatint.2016.12.004.

Seto, K. C. Sanchez-Rodriguez, R. \& Fragkias, M. (2010) The new geography of contemporary urbanization and the environment. Annual Review of Environment and Resources, Palo Alto, USA, v.35 ,n.1, p. 167-194.

šmilauer p, lepš j.(2014) Multivariate analysis of ecological data using CANOCO 5. Cambridge University Press; Cambridge http://dx.doi.org/10.1017/CBO9781139627061

Silva, R. G.; Ribeiro, C. G. (2004) Análise da degradação ambiental na Amazônia Ocidental: um estudo de caso dos municípios do Acre. Revista de Economia e Sociologia Rural, Brasília: SOBER, v. 42, n. 1, p. 91-110, 2004.

Villaça, F. (2001) Espaço intra-urbano no Brasil. São Paulo: Studio Nobel,2001.373p.

Yan, Y., Wang, C., Quan, Y., Wu, G., \& Zhao, J. (2018). Urban sustainable development efficiency towards the balance between nature and human well-being: Connotation, measurement, and assessment. Journal of Cleaner Production, 178, 67-75. DOI: 10.1016/j.jclepro.2018.01.013

Ye, B.; Cheng, M.J.; Zhang, Y.M. (2011) Suitability evaluation of development land in urban master plan. City Plan. Rev.n. 35, p. 42-44.

Zambon, I.; Benedetti, A.; Ferrara, C.; Salvati, L. (2018) Soil Matters? A multivariate analysis of socioeconomic constraints to urban expansion in Mediterranean Europe. Ecol. Econ. Amsterdam, n. 146,p. 173-183. 